\title{
Compact scattered spaces in forcing extensions
}

\author{
by
}

Kenneth Kunen (Madison, WI)

\begin{abstract}
We consider the cardinal sequences of compact scattered spaces in models where $\mathrm{CH}$ is false. We describe a number of models of $2^{\aleph_{0}}=\aleph_{2}$ in which no such space can have $\aleph_{2}$ countable levels.
\end{abstract}

All spaces considered here are assumed to be Hausdorff. The following definition summarizes the standard Cantor-Bendixson sequence:

Definition 1. For any topological space $X$ :

(1) $I(X)$ is the set of isolated points of $X$.

(2) $X^{(0)}=X$.

(3) $X^{(\alpha+1)}=X^{(\alpha)} \backslash I\left(X^{(\alpha)}\right)$.

(4) $X^{(\gamma)}=\bigcap_{\alpha<\gamma} X^{(\alpha)}$ for limit $\gamma$.

(5) $X$ is scattered if $X^{(\alpha)}=\emptyset$ for some $\alpha$; then, the least such $\alpha$ is called ht $(X)$, the height of $X$.

Equivalently, $X$ is scattered iff $I(Y) \neq \emptyset$ for all nonempty $Y \subseteq X$. If $X$ is compact scattered and nonempty, then $\mathrm{ht}(X)$ is a successor ordinal, $\delta+1$, and $X^{(\delta)}$ is finite.

Juhász, Shelah, Soukup, and Szentmiklóssy [3] study the possible values for the cardinal sequence, $\left\langle\left|I\left(X^{(\alpha)}\right)\right|: \alpha<\mathrm{ht}(X)\right\rangle$, for scattered spaces $X$. The paper [3], combined with earlier work, shows that the class of cardinal sequences obtained from the regular scattered spaces is determined by cardinal arithmetic, but this is not true for compact spaces. In particular, consider the following assertions:

(A) There is a compact scattered $X$ such that $h t(X)=\omega_{2}+1$ and $\left|I\left(X^{(\alpha)}\right)\right|=\aleph_{0}$ for all $\alpha<\omega_{2}$.

(B) $\quad\left|\left\{\alpha<\operatorname{ht}(X):\left|I\left(X^{(\alpha)}\right)\right|=\aleph_{0}\right\}\right| \leq \aleph_{1}$ for every compact scattered $X$.

2000 Mathematics Subject Classification: Primary 54G12; Secondary 03E35.

Key words and phrases: scattered space, Cantor-Bendixson sequence.

Author partially supported by NSF Grant DMS-0097881. 
Clearly (B) implies $\neg(\mathrm{A})$. Baumgartner and Shelah [1] showed that (A) is consistent with $2^{\aleph_{0}}=\aleph_{2}$, whereas Just [5] showed that $\neg(\mathrm{A})$ is consistent with $2^{\aleph_{0}}=\aleph_{2}$. Just's model was the standard Cohen real extension over a model of $\mathrm{CH}$. This result was improved by [3], which showed that (B) holds in the Cohen extension.

The argument in [3] is very specific to Cohen forcing, and they ask whether (B) holds in similar extensions, such as the one obtained by adding $\aleph_{2}$ random reals side-by-side. We show here (see Theorem 8 ) that in fact (B) does hold in this model, and also in the model obtained by adding $\aleph_{2}$ random reals in the standard way (by one measure algebra). We do not know whether (B) follows from some abstract principle, such as $C^{s}\left(\omega_{2}\right)$ described by Juhász, Soukup, and Szentmiklóssy [4]; this is also asked in [3]. However, since (B) is true in the random real model, where $C^{s}\left(\omega_{2}\right)$ is false (because there is an $\omega_{2}$-Luzin gap), it would be more interesting if one could derive (B) from some abstract principle true in both the Cohen and random extensions.

Some remarks: [3] states (B) for locally compact spaces, but that is equivalent (by taking the one-point compactification). Likewise, if (A) holds, one can remove the top level and get a locally compact $X$ of height $\omega_{2}$ such that all $I\left(X^{(\alpha)}\right)$ are countable. Under $\mathrm{CH},(\mathrm{B})$ is true, since if $\alpha$ is least with $I\left(X^{(\alpha)}\right)$ countable, then the weight of $X^{(\alpha)}$ is no more than $2^{\aleph_{0}}=\aleph_{1}$, so that $\operatorname{ht}(X)<\alpha+\omega_{2}$.

Similarly to [3], our argument assumes that (B) is false, and produces an infinite independent sequence of clopen sets, contradicting the assumption that $X$ is scattered. Our plan is to associate trees with scattered spaces, and prove (Lemma 6 ) that $\neg(\mathrm{B})$ will yield a tree of large rank, whereas (Lemma 9) in certain forcing extensions, all such trees must have small rank.

We begin with some elementary remarks on independent sequences:

Definition 2. Assume that $\nu \leq \omega$ and $K_{i} \subseteq X$ for $i<\nu$. Let $K_{i}^{0}=K_{i}$ and $K_{i}^{1}=X \backslash K_{i}$. Then $\left\langle K_{i}: i<\nu\right\rangle$ is an independent $\nu$-sequence of subsets of $X$ if $\bigcap_{i<n} K_{i}^{s(i)} \neq \emptyset$ for each finite $n \leq \nu$ and each $s \in\{0,1\}^{n}$.

Lemma 3. If $X$ is compact scattered, then there is no independent $\omega$ sequence of clopen subsets of $X$.

If $X$ is also infinite, there will be independent $n$-sequences of clopen subsets for each finite $n$. These finite sequences form a tree in the natural way, ordered by extension; the root of the tree is the empty sequence, $\emptyset$. Then by Lemma 3, this tree is well-founded - that is, it can have no infinite paths. Our proof of Theorem 8 will require a somewhat more complicated tree; we plan to use the following definition with $\gamma \leq \mathrm{ht}(X)$ and $D \subseteq X$ : 
Definition 4. Suppose that $\mathcal{C}_{\xi} \subseteq \mathcal{P}(D)$ for each $\xi<\gamma$. Define the tree $T=T_{D}\left(\left\langle\mathcal{C}_{\xi}: \xi<\gamma\right\rangle\right)$ as follows:

(1) The nodes of $T$ at level $n$ are pairs $(\vec{\xi}, \vec{H})$, where $\vec{\xi}=\left\langle\xi_{i}: i\langle n\rangle \in \gamma^{n}\right.$ is a sequence of distinct ordinals and $\vec{H}=\left\langle H_{i}: i<n\right\rangle \in(\mathcal{P}(D))^{n}$ is an independent sequence of subsets of $D$, with each $H_{i} \in \mathcal{C}_{\xi_{i}}$.

$(2)<$ denotes the usual tree order (i.e., coordinatewise extension), with the root $(\emptyset, \emptyset)$ at the top.

If $T$ is well-founded, let $\varrho=\varrho_{T}$ be its rank function, defined by $\varrho(x)=$ $\sup \{\varrho(y)+1: y \in T \& y<x\}$; also, define $\operatorname{rank}(T)=\varrho_{T}(\emptyset, \emptyset)$, and define $\operatorname{Mrank}_{D}\left(\left\langle\mathcal{C}_{\xi}: \xi<\gamma\right\rangle\right)$ to be the minimum possible value among all ordinals $\operatorname{rank}\left(T_{D}\left(\left\langle\mathcal{C}_{\xi_{\mu}}: \mu<\gamma\right\rangle\right)\right)$, where $\left\langle\xi_{\mu}: \mu<\gamma\right\rangle$ is a strictly increasing $\gamma$-sequence of ordinals less than $\gamma$.

Thus, leaf nodes (ones with no proper extension in $T$ ) will have rank 0 , and the root $(\emptyset, \emptyset)$ will have the largest rank, which we are calling $\operatorname{rank}(T)$. Then $T_{D}\left(\left\langle\mathcal{C}_{\xi_{\mu}}: \mu<\gamma\right\rangle\right)$ will always be a subtree of $T$, so its rank is $\leq$ $\operatorname{rank}(T)$; and $\operatorname{Mrank}_{D}\left(\left\langle\mathcal{C}_{\xi}: \xi<\gamma\right\rangle\right)$ is the least among the ranks of these subtrees. Note that Definition 4 does not refer to any topology on $D$. The topology arises in the following lemma:

Lemma 5. Let $\gamma$ be any limit ordinal. Assume that:

(1) $X$ is compact scattered.

(2) $E_{\xi}$, for $\xi<\gamma$, are disjoint nonempty subsets of $X$, and $\bar{E}_{\eta} \supseteq E_{\xi}$ whenever $\eta<\xi<\gamma$.

(3) Each $\mathcal{B}_{\xi}$ is a subalgebra of the clopen subsets of $X$ which separates the points of $E_{\xi}$.

Then $\operatorname{Mrank}_{X}\left(\left\langle\mathcal{B}_{\xi}: \xi<\gamma\right\rangle\right) \geq \gamma$.

Proof. Let $T=T_{X}\left(\left\langle\mathcal{B}_{\xi}: \xi<\gamma\right\rangle\right)$. It is sufficient to prove that $\operatorname{rank}(T)$ $\geq \gamma$ because every subsequence of $\left\langle E_{\xi}: \xi<\gamma\right\rangle$ has the same properties.

Call $x=(\vec{\xi}, \vec{H})$ at level $n>0$ of $T$ special if $\xi_{0}>\xi_{1}>\cdots>\xi_{n-1}$ and $E_{\xi_{n-1}} \cap \bigcap_{i<n} H_{i}^{s(i)} \neq \emptyset$ for each $s \in 2^{n}$. We prove that $\varrho(x) \geq \xi_{n-1}$ for such special nodes $x$. That will imply the desired lemma, since such special nodes clearly exist (even with $n=1$ ) for each possible value of $\xi_{n-1}<\gamma$.

The proof proceeds by induction on $\xi_{n-1}$. Thus, fix $x=(\vec{\xi}, \vec{H})$. It is sufficient to prove that for each $\eta<\xi_{n-1}, x$ has an extension of the form $y=\left(\overrightarrow{\xi^{\prime}}, \vec{H}^{\prime}\right)$, where $y$ is special, $\vec{\xi}^{\prime}=\left\langle\xi_{0}, \ldots, \xi_{n-1}, \xi_{n}\right\rangle, \xi_{n}=\eta$, and $\vec{H}^{\prime}$ extends $\vec{H}$. Then $H^{\prime}$ will be of the form $\left\langle H_{0}, \ldots, H_{n-1}, H_{n}\right\rangle$, and we need to define $H_{n}$. Since each clopen set $\bigcap_{i<n} H_{i}^{s(i)}$ meets $E_{\xi_{n-1}}$, and $\bar{E}_{\eta} \supseteq E_{\xi_{n-1}}$, each $\bigcap_{i<n} H_{i}^{s(i)}$ meets $E_{\eta}$ in an infinite set. Since $\mathcal{B}_{\eta}$ is an algebra and 
separates the points of $E_{\eta}$, we can choose $H_{n}$ so that it and its complement meet all the $2^{n}$ sets $E_{\eta} \cap \bigcap_{i<n} H_{i}^{s(i)}$.

We remark that if $\operatorname{ht}(X) \geq \gamma$, then (1) and (2) are satisfied by taking $E_{\xi}=I\left(X^{(\xi)}\right)$. If all the $E_{\xi}$ of Lemma 5 are countable, then we obtain the situation of Definition 4 with $D=\omega$ :

Lemma 6. Suppose that there are $X, \gamma$, and $E_{\xi}($ for $\xi<\gamma)$ which satisfy (1) and (2) from Lemma 5 , and in addition all $\left|E_{\xi}\right|=\aleph_{0}$. Then there are countable subalgebras $\mathcal{C}_{\xi} \subseteq \mathcal{P}(\omega)$ for $\xi<\gamma$ such that $T_{\omega}\left(\left\langle\mathcal{C}_{\xi}: \xi<\gamma\right\rangle\right)$ is well-founded and $\operatorname{Mrank}_{\omega}\left(\left\langle\mathcal{C}_{\xi}: \xi<\gamma\right\rangle\right) \geq \gamma$.

Proof. Without loss of generality, $X=\bar{E}_{0}$ (if not, replace $X$ by $\bar{E}_{0}$ ). Then we can assume that $E_{0}=\omega$. Choose $\mathcal{B}_{\xi}$ as in (3) of Lemma 5 , with $\mathcal{B}_{\xi}$ countable. Let $\mathcal{C}_{\xi}=\left\{H \cap \omega: H \in \mathcal{B}_{\xi}\right\}$. Then $\mathcal{C}_{\xi}$ is a countable subalgebra of $\mathcal{P}(\omega)$. Observe that the map $H \mapsto H \cap \omega$ is an isomorphism from the algebra of clopen subsets of $X$ into $\mathcal{P}(\omega)$; its inverse is the map $K \mapsto \bar{K}$. $\operatorname{Thus} \operatorname{Mrank}_{\omega}\left(\left\langle\mathcal{C}_{\xi}: \xi<\gamma\right\rangle\right) \geq \gamma$ follows by Lemma 5 .

We now turn to forcing extensions. As usual (see, e.g., $[2,6]$ ), a partial order $\mathbb{P}$ really denotes a triple, $(\mathbb{P}, \leq, \mathbb{1})$, where $\leq$ is a transitive reflexive relation on $\mathbb{P}$ and $\mathbb{1}$ is a largest element of $\mathbb{P}$. Then $\prod_{i \in \theta} \mathbb{P}_{i}$ denotes the product of the $\mathbb{P}_{i}$, with the natural product order. Elements $\vec{p} \in \prod_{i \in \theta} \mathbb{P}_{i}$ are $\theta$-sequences, with each $p_{i} \in \mathbb{P}_{i}$. The finite support product is given by:

Definition 7. If $\vec{p} \in \prod_{i \in \theta} \mathbb{P}_{i}$, then the support of $\vec{p}, \operatorname{supt}(\vec{p})$, is $\{i \in \theta$ : $\left.p_{i} \neq \mathbb{1}\right\}$. Moreover, $\prod_{i \in \theta}^{\text {fin }} \mathbb{P}_{i}=\left\{\vec{p} \in \prod_{i \in \theta} \mathbb{P}_{i}:|\operatorname{supt}(\vec{p})|<\aleph_{0}\right\}$.

If all $\mathbb{P}_{i}$ are countable and non-atomic, one gets the Cohen real extension, adding $\theta$ Cohen reals (note that this is the same extension for $1 \leq \theta$ $\leq \aleph_{0}$ ). To get the random real extension (see [2]), $\mathbb{P}$ is a measure algebra; for example, the measure algebra of $\{0,1\}^{\theta}$ adds $\theta$ random reals when $\theta$ is infinite. This is different from the side-by-side random real extension, which uses $\prod_{i \in \theta}^{\mathrm{fin}} \mathbb{P}_{i}$, where each $\mathbb{P}_{i}$ is the measure algebra of $\{0,1\}^{\omega}$.

The following theorem yields (B) in the extension when the ground model satisfies $\mathrm{CH}$ :

THEOREM 8. In the ground model $V$, set $\kappa=\left(2^{\aleph_{0}}\right)^{+}$. Let $\mathbb{P}$ be ccc and be either a random real extension (adding any number of random reals), or of the form $\prod_{i \in \theta}^{\text {fin }} \mathbb{P}_{i}$, where all the $\mathbb{P}_{i}$ are isomorphic and $\left|\mathbb{P}_{i}\right| \leq 2^{\aleph_{0}}$ (but $\theta$ is arbitrary). Then in the generic extension $V[G]$, the following holds: Suppose $X, \gamma$, and $E_{\xi}$, for $\xi<\gamma$, satisfy (1) and (2) of Lemma 5 , and in addition all $\left|E_{\xi}\right|=\aleph_{0}$. Then $\gamma<\kappa$. In particular, whenever $X$ is compact scattered, $\left|\left\{\alpha<\operatorname{ht}(X):\left|I\left(X^{(\alpha)}\right)\right|=\aleph_{0}\right\}\right|<\kappa$. 
To prove this, we use a lemma about ranks whose conclusion goes in the opposite direction from that of Lemma 6 :

Lemma 9. In the ground model $V$, set $\kappa=\left(2^{\aleph_{0}}\right)^{+}$. Let $\mathbb{P}$ be ccc and be either a random real extension, or of the form $\prod_{i \in \theta}^{\mathrm{fin}} \mathbb{P}_{i}$, where all the $\mathbb{P}_{i}$ are isomorphic and $\left|\mathbb{P}_{i}\right| \leq 2^{\aleph_{0}}$. Then in the generic extension $V[G]$, the following holds: If $\left\langle\mathcal{C}_{\xi}: \xi<\kappa\right\rangle$ is as in Definition 4 , with $D=\omega$, all $\left|\mathcal{C}_{\xi}\right|=\aleph_{0}$, and $T_{\omega}\left(\left\langle\mathcal{C}_{\xi}: \xi<\kappa\right\rangle\right)$ well-founded, then $\operatorname{Mrank}_{\omega}\left(\left\langle\mathcal{C}_{\xi}: \xi<\kappa\right\rangle\right)<\omega_{1}$.

Proof. We actually obtain the appropriate subsequence $\left\langle\xi_{\mu}: \mu<\kappa\right\rangle$ in $V$ by a standard thinning-out process. We first consider the case that $\mathbb{P}=\prod_{i \in \theta}^{\mathrm{fin}} \mathbb{P}_{i}$, and then comment on what needs to be changed if $\mathbb{P}$ is a random real forcing.

In $V$, for $J \subseteq \theta$, let $\mathbb{P}_{J}=\prod_{j \in J}^{\text {fin }} \mathbb{P}_{j}$, which we identify as a suborder of $\mathbb{P}$. We denote by $\mathbb{P}_{\emptyset}$ the one-element order $\{\mathbb{1}\}$. We have, for each $\xi<\kappa$, a name $\dot{\mathcal{C}}_{\xi}$ which is forced by $\mathbb{1}$ to denote a countable subset of $\mathcal{P}(\omega)$. Thus, $\dot{\mathcal{C}}_{\xi}$ is really a $\mathbb{P}_{J_{\xi}}$-name, where $J_{\xi} \subseteq \theta$ is countable. Since $\kappa=\left(2^{\aleph_{0}}\right)^{+}$, we may assume without loss of generality that the $J_{\xi}$ form a $\Delta$-system with some countable root $R$. The $\mathbb{P}_{R}$-extension of $V$ still satisfies $\kappa=\left(2^{\aleph_{0}}\right)^{+}$, so, replacing $V$ by its $\mathbb{P}_{R}$-extension, we may assume without loss of generality that $R=\emptyset$, so that the $J_{\xi}$ are disjoint. We may also assume that the $\left|J_{\xi}\right|$ are all the same cardinal $\lambda \leq \aleph_{0}$. But now we may assume that $J_{\xi}=\{\xi\}$ and $\theta=\kappa$, since we may replace the $\mathbb{P}_{i}$ by the finite support product of $\lambda$ of the $\mathbb{P}_{i}$, and simply discard the indices $i \in \theta \backslash \bigcup_{\xi} J_{\xi}$. Now, each $\dot{\mathcal{C}}_{\xi}$ is a $\mathbb{P}_{\xi}$-name. Without loss of generality, all the $\mathbb{P}_{\xi}$ are the same (since they are isomorphic), so that whenever $\pi$ is a permutation of $\kappa$, it induces a natural automorphism $\hat{\pi}$ of $\mathbb{P}$. This automorphism also applies to the $\mathbb{P}$-names, so that each $\widehat{\pi}\left(\dot{\mathcal{C}}_{\xi}\right)$ is a $\mathbb{P}_{\pi(\xi)}$-name. But $\left|\mathbb{P}_{\xi}\right| \leq 2^{\aleph_{0}}$, so there are only $2^{\aleph_{0}} \mathbb{P}_{\xi^{-}}$ names for countable subsets of $\mathcal{P}(\omega)$, so we may assume that $\widehat{\pi}\left(\dot{\mathcal{C}}_{\xi}\right)$ is always the name $\dot{\mathcal{C}}_{\pi(\xi)}$.

Still in $V$, we have a name $\dot{T}$ for the tree $T_{\omega}\left(\left\langle\dot{\mathcal{C}}_{\xi}: \xi<\kappa\right\rangle\right)$. We also have a name $\dot{\varrho}$ for the rank function. Whenever $\vec{\xi} \in \kappa^{<\omega}$ is a sequence of distinct ordinals, let

$$
S_{\vec{\xi}}=\{\sigma: \exists p \in \mathbb{P}[p \Vdash[\exists \vec{H}[(\vec{\xi}, \vec{H}) \in \dot{T} \& \dot{\varrho}(\vec{\xi}, \vec{H})=\sigma]]]\} .
$$

Then each $\left|S_{\vec{\xi}}\right| \leq \aleph_{0}$ (since $\dot{\mathcal{C}}_{\xi}$ is forced to be countable), and $S_{\vec{\xi}}$ only depends on the length of $\vec{\xi}$ (using the automorphisms; note that in Definition 4, the tree order does not depend on the ordering of the ordinal $\gamma$ ). Thus, if we set $S=\bigcup\left\{S_{\vec{\xi}}: \vec{\xi} \in \kappa^{<\omega}\right\}$, then $|S| \leq \aleph_{0}$. Since $S$ must also be an initial segment of the ordinals, it is forced by $\mathbb{1}$ that $\operatorname{rank}(\dot{T})<\omega_{1}$. 
Now, in the random real case, we may assume (in $V$ ) that the elements $p \in \mathbb{P}$ are the Baire subsets of $2^{\theta}$ of positive measure. The order $\leq$ is just $\subseteq$, and $\mathbb{1}=2^{\theta}$. If $J \subseteq \theta$, we let $\mathbb{P}_{J}$ be the set of elements $p \in \mathbb{P}$ such that $J$ is a support of $p$ (i.e., $\forall f, g \in 2^{\theta}[f\lceil J=g\lceil J \rightarrow[f \in B \leftrightarrow g \in B]]$ ). Every Baire subset of $2^{\theta}$ has a countable support, so that each $\dot{\mathcal{C}}_{\xi}$ is a $\mathbb{P}_{J^{-}}$ name for some countable $J$. The same countable $\Delta$-system argument lets us assume that, without loss of generality, each $\dot{\mathcal{C}}_{\xi}$ is a $\mathbb{P}_{J_{\xi}}$-name, where $J_{\xi}=\{\omega \cdot \xi+n: n \in \omega\}$. Now, $\widehat{\pi}$ is the automorphism of $\mathbb{P}$ which arises from permuting coordinate $\omega \cdot \xi+n$ to coordinate $\omega \cdot \pi(\xi)+n$. The proof that $\mathbb{1} \Vdash \operatorname{rank}(\dot{T})<\omega_{1}$ is the same as before.

Proof of Theorem 8. The fact that $\gamma<\kappa$ is immediate from Lemmas 6 and 9. For the "in particular": If that failed, we could let $\left\langle\alpha_{\xi}: \xi<\kappa\right\rangle$ be an increasing sequence of ordinals with each $\left|I\left(X^{\left(\alpha_{\xi}\right)}\right)\right|=\aleph_{0}$; then setting $\gamma=\kappa$ and $E_{\xi}=I\left(X^{\left(\alpha_{\xi}\right)}\right)$, we would have a contradiction.

Acknowledgements. We would like to thank István Juhász for some useful comments on the original draft of this paper.

\section{References}

[1] J. E. Baumgartner and S. Shelah, Remarks on superatomic Boolean algebras, Ann. Pure Appl. Logic 33 (1987), 109-129.

[2] T. Jech, Set Theory, third millennium edition, Springer, Berlin, 2003.

[3] I. Juhász, S. Shelah, L. Soukup, and Z. Szentmiklóssy, Cardinal sequences and Cohen real extensions, Fund. Math. 181 (2004), 75-88.

[4] I. Juhász, L. Soukup, and Z. Szentmiklóssy, Combinatorial principles from adding Cohen reals, in: Logic Colloquium '95 (Haifa), Springer, Berlin, 1998, 79-103.

[5] W. Just, Two consistency results concerning thin-tall Boolean algebras, Algebra Universalis 20 (1985), 135-142.

[6] K. Kunen, Set Theory, North-Holland, Amsterdam, 1980.

Department of Mathematics

University of Wisconsin

Madison, WI 57306, U.S.A.

E-mail: kunen@math.wisc.edu

Web: http://www.math.wisc.edu/ ${ }^{\sim}$ kunen

Received 12 August 2004;

in revised form 21 December 2004 